\title{
El diseño de criterios de manejo del paciente diabético como estrategia de garantía de calidad
}

\author{
Designing criteria to handle diabetes patients \\ as a strategy for quality assurance
}

Armando Martínez Ramírez 1

César Gray Santacruz 2

Javier Eduardo García de Alba 3

Sandra Reyes Fraustro 4

1 Hospital General Regional
no 46, Instituto Mexicano
del Seguro Social, Jalisco,
México.
2 Hospital General de Zona
no 14, Instituto Mexicano
del Seguro Social, Jalisco,
México.
3 Universidad de
Guadalajara, Jalisco, México.
4 Subjefatura de
Investigación Epidemi ológica
y de Servicios de Salud,
Instituto Mexicano del
Seguro Social, Jalisco,
México.
Dirección:
Privada Colomos 1.883,
Depto. 201 -c,
Colomos Providencia,
Guadalajara, Jalisco, México,
C. P. 44.620
A bstract This study was carried out at the General Hospital of Zone № 14 of the IMSS in Jalisco, Mexico, with the goal of designing standardized criteria to handle hospitalized diabetes patients, as a strategy for quality assurance, through the participation of a panel of experts in internal medicine from the hospital. Participation by the attending physician in designing criteria generates a sense of bel onging in the staff and makes adherence and application easier. The suggested criteria are subject to further improvement and are not intended for universal application, but to the extent that they have been useful in patient care they have far surpassed theauthors' expectations when the goals of this research were originally established.

Key words Health Services; Health Evaluation; Diabetes Mellitus

Resumen El presente estudio se realizó en el Hospital General deZona no 14 en Jalisco, México, con el objetivo de diseñar criterios estandarizados de manejo del paciente diabético en los servici os de hospital ización, como una estrategia de garantía de cali dad, a través de la partici pación de un panel de expertos del servicio de medicina interna del propio hospital. La participación del médico responsable del proceso de la atención médica en el diseño de los criterios genera en el personal un sentido de pertenencia y facilita su adherencia y aplicación. Ciertamente, los criterios sugeridos son perfectibles y de ninguna manera se pretendesu aplicación universal, si de al guna manera resultan útiles para quienes atienden estos pacientes, rebasaría con mucho los objetivos que los autores se plantearon en la planeación dela investigación.

Palabras clave Servicios deSalud; Evaluación en Salud; Diabetes Mel litus 


\section{Introducción}

La Organización Panamericana de la Salud (OPS) menciona que el desarrollo de Programas de Garantía de Calidad es una necesidad en términos de eficiencia y una obligación en términos éticos y morales (Paganini \& De Moraes, 1992).

Para Donabedian (1986a; 1986b; 1986c), existen dos elementos centrales para garantizar la calidad: monitoría y un adecuado diseño del sistema de salud. En cambio, para Ruelas \& Reyes (1990), son cinco los el ementos fundamentales que permiten garantizarla: evaluación, monitoreo, diseño, desarrollo y cambio organizacional. Asimismo, se han propuesto tres tipos de programas de garantía de calidad: centralizados, descentralizados no participativos y descentralizados participativos, basados en el grado de participación de los diferentes actores del proceso de la atención médica (Ruelas \& Frenk, 1989).

Por otra parte, existen varios métodos para diseñar los criterios y están dares para garantizar la calidad; tres son los más connotados: el propuesto por Donabedian, cuyas aportaciones más importantes son la descripción detallada de las características de los criterios, los elementos para evaluarlos (validez) y los procedimientos para su formulación (Donabedian, 1986a; 1986b; 1986c; 1992); el referido por Jackson (1992), y diseñado por un panel de expertos quienes proponen una serie de atributos divididos en dos categorías, los atributos sustantivos y estructural es y los de implementación y proceso; y, finalmente el diseñado por Vidal \& Reyes (1993a; 1993b), quienes enfatizan la necesidad de que los criterios cumplan con los requisitos de validez, objetividad, sensibilidad y especificidad, dándole un valor (estándar), y por supuesto diseñar un sistema de información que asegure la captura, el procesamiento y el análisis de datos, regido por los principios de participación, sistematización, la implantación secuencial, la multiplicación y la simplificación, lo que favorecería a la comprensión de la estrategia por parte del personal y el apoyo de los niveles directivos.

El presente estudio se realizó en el Hospital General deZona (HGZ) no 14, Instituto Mexicano del Seguro Social (IMSS) en Jalisco, M éxico, con el objetivo de diseñar criterios estandarizados de manejo del paciente diabético como una estrategia de garantía de calidad, a través de la participación de un panel de expertos del servicio de medicina interna del propio hospital.

\section{Material y métodos}

El Instituto Mexicano del Seguro Social (IMSS), es un sistema de salud gubernamental descentralizado y constituye la organización mexicana de seguridad social más grande ya que proporciona atención médica a cerca del $50 \%$ de la población.

EI HGZ no 14 es una unidad de segundo nivel del sistema IMSS en Jalisco, tiene una capacidad física instalada de 226 camas y atiende una demanda potencial de 394,951 derechohabientes, cuenta con 24 especialidades, entre ellas medicina interna. La patología que más impacta en la solicitud y utilización de servicios es la diabetes mellitus, ya que, por ejemplo, en 1993, se otorgaron 1324 consultas con tasa de 3,35 por 1000 usuarios; fue la principal causa de egreso hospitalario con 1092 casos y una tasa de 2,76 por 1000 pacientes hospitalizados; además, fue la primera causa de defunción, con 92 muertes, correspondiéndole el 17,43\% del total de fallecimientos que ocurrieron entre los pacientes que ingresaron al hospital.

En abril de 1994, se inició en este hospital el presente estudio, con previa autorización y consentimiento de los directivos del hospital y del Comité Local de Investigación. La estrategia que se utilizó fue eminentemente participativa y la incorporación de los expertos al grupo de trabajo fue de carácter voluntario.

Se decidió trabajar en jornadas semanales de dos horas, con el objeto de que la investigación no interfiriera en las actividades habituales del personal médico, y durante las primeras 8 sesiones se trataron temas sobre: aspectos conceptuales de la calidad del modelo industrial y el modelo médico, sus diferencias y similitudes; análisis de la situación institucional en relación a la calidad a través de un ejercicio metodológico que resultó de mucha utilidad, sobre todo, para definir el problema de estudio; posteriormente se analizaron los programas de garantía de calidad revisando con detalle los siguientes elementos: evaluación, monitoría, diseño, desarrollo y cambio organizacional. A partir de julio de 1994 se inició el diseño operativo de los criterios, fase que terminó en septiembre del mismo año.

Los criterios incluyen: la identificación del paciente; el interrogatorio, que a su vez se subdivide en signos y síntomas principales y antecedentes. En este subcapítulo se propone investigar: tabaquismo y alcoholismo, conocidos factores de riesgo de la enfermedad; exploración física que incluye revisión oftalmológica y de los pies; complicaciones; confirmación laboratorial; estudios de gabinete, sobre todo en 
mayores de 45 años (P.A. Tórax y electrocardiograma); clasificación de la diabetes; dieta; ejercicio y medidas generales; educación/información (este rubro lo consideramos sumamente valioso, no sólo para el adecuado control del paciente diabético, sino también para evitar las complicaciones tempranas y tardías, que con frecuencia conducen estos pacientes a un grave deterioro de la calidad de vida y/o a la muerte); y finalmente el tratamiento.

Además, se incorporan indicadores de resultados como son: complicaciones hospitalarias (infecciosas y no infecciosas), los criterios de alta, la estancia hospitalaria y los reingresos dentro de los primeros 30 días de que el paciente salió del hospital.

La ponderación de los ITEM (estandarización) se hizo tomando en cuenta los niveles de prevención de Leavell y Clark, teniendo más valor la prevención primaria (3) que la secundaria (2) y la terciaria (1); y dentro de cada una de estas, las correspondientes a la promoción de la salud (3) y la protección específica (2), dentro del primer nivel de prevención; diagnóstico oportuno (3), tratamiento precoz (2) y limitación del daño (1), dentro del segundo nivel; y la rehabilitación física, moral y social con el mismo valor ponderal (2), en el tercer nivel de prevención. La lógica de este razonamiento es que entre más oportuna sea la intervención, mejores resultados se obtienen durante el proceso de la atención médica, por lo tanto deben tener mayor puntaje.

El criterio estadístico utilizado para ubicar las categorías de calidad fue el de percentiles, sobre la base de que no necesariamente la curva de distribución de frecuencias a obtenerse fuera normal. Así entonces, utilizamos los percentiles 90, 75, 50 y 25 como límites de clase para los valores asignados como categorías de calidad: Excelente >Pc 90; $\varangle$ Buena>Pc 75; $<$ Suficiente>Pc 50; 4 nsuficiente>Pc 25<Inadecuada.

Se decidió trabajar con dos grupos: el Grupo 1, integrado por 20 expedientes clínicos de pacientes que habían egresado con diagnóstico de diabetes mellitus tipo II (evaluación basal), y el Grupo 2, conformado por 50 pacientes hospitalizados con el mismo diagnóstico. Los valores de las puntuaciones obtenidas, correspondientes a estos percentiles, fueron:

\begin{tabular}{llc}
\hline & Grupo 1 & Grupo 2 \\
\hline PC 90 & 38 & 97 \\
PC 75 & 33 & 87 \\
PC 50 & 29.5 & 77 \\
PC 25 & 27 & 70 \\
\hline
\end{tabular}

En cualquier proceso de evaluación es necesario asignarle un juicio de valor a la norma de operación. Aguirre propone una alternativa para lograr una escala de evaluación de los elementos de la atención médica, eminentemente subjetivos, al determinar parámetros cualitativos que permitan discriminar con claridad los diferentes rangos de calidad, sin imbricarse y sin permitir sesgos, condicionados por prejuicios, que pueden ser determinados por un sí o no absolutos (Aguirre, 1993). Con ligeras modificaciones utilizaremos esta escala que incluye las siguientes categorías:

Excel ente: Logro del objetivo de la atención médica y de la satisfacción del paciente como resultado de una atención oportuna y un proceso de atención correctamente realizado, en un ambiente confortable, con trato personalizado y amable, con una comunicación abierta con el paciente, que conduzcan a su satisfacción plena por los servicios recibidos.

Buena: Logro del objetivo de la atención médica y de la satisfacción del paciente como resultado de una atención oportuna y un proceso de atención correctamente realizado.

Suficiente: Oportuna realización del proceso de atención, que permita conseguir la solución integral del problema, el máximo factible de mejoría y la ausencia de secuelas o complicaciones sobre la premisa de un proceso bien llevado a cabo.

Insuficiente: Realización correcta del proceso, pero fuera del momento oportuno para llevarlo a cabo, con logro parcial del objetivo, pueden presentarse complicaciones que prolonguen la estancia hospitalaria.

Inadecuado: Se presenta por la realización incorrecta de la atención médica, por un proceso de atención llevado a cabo con carencia de oportunidad, con ignorancia o aplicación deficiente de normas, conocimientos médicos o tecnología vigente que conduzcan a resultados negativos, con daño al paciente por complicaciones, secuelas o inclusive defunción.

Una vez que se diseñaron los criterios y su ponderación, se los presentaron para su validación a un grupo de especialistas de otros hospitales de la institución, mismos que, previa revisión, discusión y ligeras modificaciones, consideraron que su aplicación sistemática podría mejorar la calidad de la atención médica del paciente diabético. 


\section{Resultados}

Se realizó una evaluación basal utilizando los mismos criterios, para lo cual se tomaron al azar 20 expedientes clínicos de pacientes que habían ingresado con diagnóstico de diabetes mellitus tipo II no insulino dependiente (DM NID) y que egresaron durante los meses de septiembre y octubre de 1994.

En 4 de los 20 pacientes (20\%), la atención, de acuerdo a la escala de calidad, fue inadecuada, ya que la puntuación obtenida fue $<27$; seis casos (30\%) se calificaron con calidad insuficiente (27-29.5); en cinco pacientes (25\%) se consideró la atención suficiente (29.5-33); en cuatro pacientes (20\%) la atención se catalogó como buena (33-38), y sólo en un caso (5\%) la atención fue excelente $>38$. En este grupo se presentaron 3 complicaciones hospitalarias (15\%), dos de tipo infeccioso - las dos infecciones de vías urinarias (IVU) - y un sangrado de tubo digestivo alto (STDA). El total de días de estancia hospitalaria de los 20 pacientes fue de 158 , con un promedio de 7,9 . Se presentaron también 3 reingresos (15\%), dentro de los primeros 30 días después del egreso, aunque es importante consignar que uno de ellos fue por agudización de una neumopatía obstructiva crónica, otra por neuropatía, pero también por complicaciones de una cirrosis hepática, y la tercera por insuficiencia renal crónica (IRC).

Para la evaluación posterior a la estrategia educativa se seleccionaron 50 pacientes al azar de los diabéticos tipo II hospitalizados en el servicio de medicina interna, con los cuales se obtuvieron los siguientes resultados: en 12 pacientes (24\%) la atención fue inadecuada, es decir, la puntuación fue de $<70 ; 13$ casos (26\%) se ubicaron con calidad insuficiente (70-77); en 12 de los pacientes (24\%) la evaluación fue de atención suficiente (77-87); 8 casos (16\%) tuvieron una atención buena (87-97), y atención excelente la obtuvieron 5 pacientes (10\%) ya que los puntos alcanzados fueron de $>97$. Las complicaciones en este grupo se presentaron en 8 pacientes (16\%), sólo que en tres pacientes se presentaron 2 complicaciones y uno presentó 3 eventos durante su hospitalización. Los tipos de complicaciones fueron 5 no infecciosas, todas trombosis venosa, y 8 de carácter infeccioso: cuatro IVU, dos úlceras por presión, una infección de catéter (Tenkoff) y una úlcera en prepucio. El promedio de estancia hospitalaria en estos pacientes fue de 8,5 días, aunque es necesario señalar que en esta serie de pacientes hubo gran dispersión de la media, ya que dentro de la muestra entraron un pa- ciente con un día de estancia y uno con 42 días, impactando, sobre todo éste último, en el promedio de días de estancia en el hospital. Además, se registraron cinco reingresos (10\%) entre estos pacientes: tres por IRC, 1 por pie diabético y otro por cirrosis hepática.

Es importante señalar que los resultados no son generalizables porque se utilizó una muestra insuficiente, ya que sólo se pretendía pilotear el protocolo de manejo de los pacientes diabéticos, por lo tanto, es indispensable manejar muestras estadísticamente representativas que permitan aumentar la validez interna y externa de los resultados.

\section{Discusión}

Actualmente, existe un crecimiento vertiginoso del interés por la calidad de la atención a la salud en México. En muchos otros países, cuyo origen es probablemente una mayor conciencia de que, en el mundo contemporáneo, la calidad se ha convertido en un requisito indispensable de sobreviviencia económica. Para algunos afortunadamente también, de responsabilidad social y de integridad moral (Ruelas \& Zurita, 1993).

En una búsqueda intencionada de antecedentes de estudios en los que se han utilizado criterios "explícitos" de calidad (1964, 1984), se señala que la corriente de estudios que usan las condiciones de salud como forma de medir la calidad inicia con la obra de Ernest Amory Codman en 1916. En1956, Paul Lembcke maneja la premisa de que las normas de la práctica médica deben expresarse en criterios explícitos y ésta fue la piedra angular en la que Lembcke construyó lo que esperaba que fuera la "Ciencia" de la evaluación (Donabedian, 1988).

En México, desde 1918 se enfatiza la trascendencia del procedimiento para garantizar y promover una atención médica de calidad. Hacia 1956, el Hospital de “La Raza” inicia en forma extraoficial la revisión del expediente clínico y, en 1957, se formó la Comisión de Supervisión Médica del IMSS (Aguirre, 1990).

Ya para 1985, se plantea el concepto de la epidemiología de la calidad, que permite describir la distribución de la calidad: primero, en quienes proporcionan la atención, cuyo principal hallazgo es la insuficiencia generalizada del desempeño relativo a las normas (criterios estandarizados de diagnóstico y tratamiento) ostensibles en la práctica médica, aunada a grandes variaciones entre distintos médicos, entre instituciones y aún entre áreas geográficas; y la 
segunda faceta, entre los beneficiarios de la atención médica (Donabedian, 1986a; 1986b; 1986c).

Por otro lado, existen diversos estudios de investigación (Laffel \& Berwick, 1992) que demuestran que los eventos adversos en la atención médica se presentan en gran medida (28\%) por la variación en los procedimientos clínicos.

Por lo antes expuesto, se hace cada vez más necesario el diseño y la utilización de criterios (algoritmos) estandarizados de manejo de los pacientes (Guiscafré et al., 1988), en cuyo diseño participen los responsables del proceso de la atención médica, lo que genera en el personal un sentido de pertenencia y facilita su adherencia y aplicación. Además, se requiere de una cultura organizacional orientada al mejoramiento continuo de la calidad, y del apoyo de procesos educativos participativo-creativos, y de la investigación en sistemas y servicios de salud, que es la que más rápidamente da res- puesta a las necesidades y demandas sociales.

Sabemos que esto implica un proceso, tal vez, largo de capacitación y motivación sobre técnicas y estrategias de garantía de calidad que conduzcan a resultados objetivos. Todo esto requiere, además, incorporar al proceso a la investigación cualitativa y cuantitativa, que permita no sólo monitorizar la calidad, sino también la efectividad y la eficiencia de los esfuerzos por garantizar la calidad y que valide científicamente los resultados con un balance positivo en relación a costo/beneficio y costo/ efectividad.

Seguramente que la lista de criterios propuesta es perfectible y de ninguna manera pretende su aplicación universal, solamente se plantea como una estrategia de garantía de calidad, y si de alguna manera resulta útil para el personal que maneja este tipo de pacientes, rebasaría con mucho los objetivos que los autores se plantearon en la planeación de este estudio de investigación.

\section{Referencias}

AGUIRRE, G. H., 1990. Evaluación de la calidad de la atención médica. Expectativas de los pacientes y de los trabajadores de las unidades médicas. Salud Pública de México, 32:170-180.

AGUIRRE, G. H., 1993. Calidad de la Atención Médica. Conferencia Interamericana de Seguridad. Secretaría General, Comisión Americana Médico Social. Serie de Estudios, (México, D.F.) 2:38-39.

DONABEDIAN, A., 1986a. Quality assurance in our health care system. Quality Assurance Utilization Review, 1:37-68.

DONABEDIAN, A., 1986b. Criteria and standars for quality assessment and monitoring. Quality Review Bulletin, 9:99-108.

DONABEDIAN, A., 1986c. La investigación sobre la calidad de la atención médica. Salud Pública de México, 28:221-229.

DONABEDIAN, A., 1988. Veinte años de investigación en torno a la calidad de la atención médica, 19641984. Salud Pública de México, 30:202-215.

DONABEDIAN, A., 1992. Defining and measuring the quality of health care. In: Assessing Quality Health Care. Perspectives for Clinicians (R. P. Wenzel, ed.), pp. 42-64. Baltimore: Williams \& Wilkins.

GUISCAFRÉ, H. et al., 1988. Evaluación de una estrategia dirigida a médicos familiares para incrementar el uso de la hidratación oral y disminuir el de los antimicrobianos y dietas restrictivas. Archivos deInvestigacción Médica Mex., 19:395-407.
JACKSON, M. M., 1992. Setting standars. In: Assesing Quality Health Care. Perspectives for Clinicians (R. P. Wenzel, ed.), pp. 65-85. Baltimore: Williams $\&$ Wilkins.

LAFFEL, G. \& BERWICK, D., 1992. Quality in Health Care. Journal of the American Medical Association. 268:407-409.

PAGANINI, J. M. \& DE MORAES, N. H., 1992. La garantía de calidad. Acreditación de Hospitales para América Latina y el Caribe. Washington, D.C: Organización Panamericana de la Salud. Federación Latinoamericana de Hospitales.

RUELAS, B. E. \& FRENK, J., 1989. Frame work for the analysis of quality assurance in transition: The case in México. Australian Clinical Review, 9:916.

RUELAS, B. E. \& REYES, Z. H., 1990. Círculos de calidad como estrategia de un programa de garantía de calidad de la atención médica en el instituto nacional de perinatología. Salud Pública deMéxiCO, 32:207-220.

RUELAS, B. E. \& ZURITA, G. B., 1993. Nuevos horizontes de la calidad de la atención a la salud. Salud Pública de México, 35:235-237.

VIDAL, P. L. M. \& REYES, Z. H., 1993. Diseño de un sistema de monitoría para el desarrollo de programas de garantía de calidad. Salud Pública de Mé xico, 35:326-331. 